\title{
Discovery of a quasi-periodic oscillation in the ultraluminous $X$-ray source IC 342 X-1: XMM-Newton results
}

\author{
V. K. Agrawal` and Anuj Nandi \\ Space Astronomy Group, SSIF/ISITE Campus, ISRO Satellite Centre, Outer Ring Road, Marathahalli, Bangalore 560037, India
}

Accepted 2014 October 29. Received 2014 October 29; in original form 2013 December 27

\begin{abstract}
We report the discovery of a quasi-periodic oscillation (QPO) at $642 \mathrm{mHz}$ in an XMM-Newton observation of the ultraluminous X-ray source (ULX) IC 342 X-1. The QPO has a centroid at $v_{\mathrm{QPO}}=642 \pm 20 \mathrm{mHz}$, a coherence factor $Q=11.6$ and an amplitude (rms) of 4.1 per cent with significance of 3.6 $\sigma$. The energy dependence study shows that the QPO is stronger in the energy range $0.3-5.0 \mathrm{keV}$. A subsequent observation ( $6 \mathrm{~d}$ later) does not show any signature of the QPO in the power-density spectrum. The broad-band energy spectra $(0.3-40.0 \mathrm{keV})$ obtained by quasi-simultaneous observations of XMM-Newton and NUSTAR can be described well by an absorbed diskbb plus cutoffpl model. The best-fitting spectral parameters are powerlaw index $(\Gamma) \sim 1.1$, cut-off energy $\left(E_{\mathrm{c}}\right) \sim 7.9 \mathrm{keV}$ and disc temperature $\left(k T_{\text {in }}\right) \sim 0.33 \mathrm{keV}$ where the QPO is detected. The unabsorbed bolometric luminosity is $\sim 5.34 \times 10^{39} \mathrm{erg} \mathrm{s}^{-1}$. Comparing this with the well-known X-ray binary GRS 1915+105, our results are consistent with the mass of the compact object in IC $342 \mathrm{X}-1$ being in the range $\sim 20-65 \mathrm{M} \odot$. We discuss the possible implications of our results.
\end{abstract}

Key words: accretion, accretion discs - black hole physics - X-rays: binaries - X-rays: individual: IC 342 X-1.

\section{INTRODUCTION}

Ultraluminous X-ray sources (ULXs) are off-nuclear point X-ray sources in nearby galaxies with isotropic luminosities $>10^{39} \mathrm{erg} \mathrm{s}^{-1}$ (see Feng \& Soria 2011 for a recent review). Since their discovery more than 30 years ago (Long \& Speybroeck 1983; Fabbiano \& Trinchieri 1987; Fabbiano 1988, 1989), the true nature of ULXs has remained a mystery. Early ASCA and XMM-Newton observations revealed that most ULXs should contain accreting black holes (Kubota et al. 2001; Sutton, Roberts \& Middleton 2013). Recently, Motch et al. (2014) reported a firm upper limit of $<15 \mathrm{M}_{\odot}$ on the mass of a black hole in a ULX, suggesting that most ULXs are indeed stellar-mass black holes. However, the discovery of X-ray pulsations (Bachetti et al. 2014) in one of the ULXs in galaxy M82 suggests that ULXs may also be powered by accretion on to magnetized neutron stars. In ULXs, mass estimates of the compact objects remain highly debatable because no dynamical measurement has been possible yet. However, recent optical/UV observations reveal that ULXs in M101 (Liu et al. 2013) and NGC 7793 (Motch et al. 2014) harbour stellar-mass black holes.

Since luminosities of ULXs exceed the Eddington rate for a $10-\mathrm{M}_{\odot}$ black hole, it has been suggested that ULXs might harbour intermediate-mass black holes (IMBHs) with masses in the

^E-mail: vivekag@isac.gov.in range $10^{2}-10^{4} \mathrm{M}_{\odot}$ (Colbert \& Mushotzky 1999; see also Pasham, Strohmayer \& Mushotzky 2014). Other popular models proposed to explain the ultraluminous nature of ULXs are (1) normal X-ray binaries (XRBs) accreting at super-Eddington rate (Begelman 2002) or (2) XRBs accreting at sub-Eddington rate with beamed emission (Reynolds et al. 1997; King 2002; Begelman, King \& Pringle 2006). However, the beaming scenario suffers from several difficulties, e.g. a lack of evidence for radio jets in ULXs (see Feng \& Soria 2011) and the presence of a strong quasi-periodic oscillation (QPO) in M82 X-1 (Strohmayer \& Mushotzky 2003). Recently, Gladstone, Roberts \& Done (2009) suggested a new accretion state named the ultraluminous state, implying a super-Eddington accretion rate for ULXs. Based on early observations with XMM-Newton, some ULX spectra $(0.3-10.0 \mathrm{keV})$ were modelled with a simple phenomenological model (i.e. disc emission and power-law component: Miller et al. 2003; Miller, Fabian \& Miller 2004) with a characteristic disc temperature of $k T_{\text {in }} \sim 0.1-0.5 \mathrm{keV}$. The presence of a cool disc component and very high luminosities, which indeed triggered the hypothesis that ULXs may contain IMBHs, may not be a valid prescription to explain most of the X-ray observational features. Recent studies (see Gladstone et al. 2009 for details) showed that the energy spectra can be well described by an optically thick corona ( $\tau \sim 5$-30, whereas $\tau \sim 1$ for the corona seen in Galactic black hole binaries) coupled with an accretion disc. In addition, the presence of a high-energy curvature $(>3.0 \mathrm{keV})$, not seen in Galactic black hole binaries (Remillard \& McClintock 2006) accreting at a 
sub-Eddington rate, is now considered as one of the ULX spectral signatures (Stobbart, Roberts \& Wilms 2006; Gladstone, Roberts $\&$ Done 2009). Furthermore, combining both spectral and temporal variabilities, Sutton et al. (2013) classified three spectral regimes for ULXs, namely the broadened disc, hard ultraluminous and soft ultraluminous classes. Recently, Pintore et al. (2014) investigated a larger sample of observations in order to study the spectral evolution with a different approach (i.e. based on colour-colour and hardness-intensity diagram analysis). All these studies corroborate further the idea of a new accretion state (i.e. the ultraluminous state) for ULXs (Roberts 2007; Gladstone et al. 2009).

The study of short-term variability may also put constraints on the various accretion models in ULXs (see Pasham et al. 2014). In a few ULXs (M82 X-1, NGC 5408 X-1, NGC 6946 X-1, M82 $\mathrm{X} 42.3+59)$, QPOs have been detected in the frequency range $\sim 3-$ $200 \mathrm{mHz}$ (Strohmayer \& Mushotzky 2003; Dewangan, Titarchuk \& Griffiths 2006a; Strohmayer et al. 2007; Rao, Feng \& Kaaret 2010; Feng, Rao \& Kaaret 2010; Pasham \& Strohmayer 2012). A QPO around $200 \mathrm{mHz}$ has been reported in Holmberg X-1 (Dewangan, Griffiths \& Rao 2006b), but was not confirmed later (see Heil, Vaughan \& Roberts 2009). Recently, Pasham et al. (2014) reported twin-peak QPOs in M82 X-1 at frequencies of 3.32 and $5.07 \mathrm{~Hz}$. Several ULXs show short-term variability with (or without) the presence of QPOs in the power spectra, whereas variability is found to be completely suppressed in some sources (Heil et al. 2009). It has been suggested that short-term variability in the ultraluminous state can be produced by variable obscuration due to clumpy winds (see Middleton et al. 2011; Sutton et al. 2013).

IC $342 \mathrm{X}-1$ is a ULX in the nearby spiral galaxy IC 342 at a distance of 3.3 Mpc (Saha, Claver \& Hoessel 2002). The source was discovered by the Einstein satellite (Fabbiano \& Trinchieri 1987). This source was also detected by ROSAT in the ultraluminous state (Bregman, Cox \& Tomisaka 1993; Roberts \& Warwick 2000). ASCA observations of the source taken during 1993 and 2000 revealed spectral transitions from a high/soft to a low/hard spectrum (Kubota et al. 2001). The analysis carried out using Suzaku, XMM-Newton, Chandra and Swift revealed two different power-law (PL) states in this source (Yoshida et al. 2013): a low-luminosity PL state and a high-luminosity PL state. Recently, Marlowe et al. (2014) reported a clear change in a recent Chandra spectrum with a much softer spectrum than seen in all previous observations and it has been modelled with standard accretion-disc emission. The source has also been detected in the radio using the Very Large Array (VLA), but the presence of a compact radio jet was not confirmed in the high spatial resolution data as observed with very-long-baseline interferometry (VLBI: Cseh et al. 2012).

In the present work, we focus on the recent quasi-simultaneous observations of IC 342 X-1 made by XMM-Newton and NUSTAR in 2012 August. Recently, Rana et al. (2014) also analysed the same data sets, in order to understand the broad-band spectral nature of the source. From our detailed analysis (see Sections 2 and 3), we report the detection of a QPO in IC 342 X-1, along with the spectral properties of the source.

\section{OBSERVATIONS AND DATA REDUCTION}

XMM-Newton observed IC 342 X-1 six times between 2001 and 2012. We use the data obtained on 2012 August 11 with an exposure time of $55 \mathrm{ks}$ (Obs-1) and on 2012 August 17 for a total exposure of $50 \mathrm{ks}$ (Obs-2). The previous data sets (i.e. the initial four observations) have been analysed by Yoshida et al. (2013, see Pintore et al. 2014 for a recent analysis). The 2012 observations were carried out in PrimeFullWindow mode with a time resolution of $73.35 \mathrm{~ms}$. Data reduction is performed using SCIENCE ANALYSIS SYSTEM (SAS) Version 12.0.1 and the recent calibration data set. We create the calibrated event files using SAS task epchain. We extract the light curve from the entire chip in the 10.0-15.0 keV band and then generate the good time interval (gti) file using the selection criteria (rate $<=3 \times$ the mean of the 10.0-15.0 keV light curve). We select EPIC-pn events with PATTERN $<=4$ and FLAG $==0$. We use a circular region of 40 arcsec centred at the source position to extract the source events. A circular region of similar size away from the source position is used to extract the background events. We apply the gti filter while creating the light curves in the $0.3-10.0 \mathrm{keV}, 0.3-5.0 \mathrm{keV}$ and 5.0 $10.0 \mathrm{keV}$ bands for Obs- 1 and Obs-2. We find that the above filtering process removes the large background flares at the end of the observations. We also observe that the filtering process produces two data gaps of 500 and $300 \mathrm{~s}$ in the light curves of Obs-1, corresponding to two short flares, and a 300-s data gap in the light curves of Obs-2.

The tasks rmfgen and arfgen are used to create a response matrix file (rmf) and ancillary response file (arf). The spectra are grouped to give a minimum of 25 count bin $^{-1}$.

NUSTAR also observed the source on 2012 August 10 for a total exposure time of $98.6 \mathrm{ks}$ and on 2012 August 16 for a total exposure of $127.3 \mathrm{ks}$. We use the most recent NUSTAR analysis software distributed with HEASOFT version 6.15 and the latest calibration files (version 20131007) for reduction and analysis of the NuSTAR data. We use the task nupipeline to generate calibrated and screened event files. A circular region of 30 arcsec centred at the source position is used to extract the source events. Background events are extracted from a circular region of the same size away from the source. The task nuproduct is used to generate the spectra and response files. The spectra are grouped to give a minimum of 25 count bin ${ }^{-1}$.

\section{ANALYSIS AND RESULTS}

The background-subtracted light curves of IC 342 X-1 observed with XMM-Newton at two different epochs (Obs-1 \& Obs-2) are shown in Fig. 1 with effective exposure times (which resulted after removing the high background intervals) of 43.5 and $37.5 \mathrm{ks}$ respectively. The average count rate for Obs- 1 is $0.40 \pm 0.02$ and that for Obs-2 is $0.51 \pm 0.02$. A variability of about 20 per cent is evident in both observations on a time-scale of a few thousand seconds. The average count rates between two observations also vary by $\sim 30$ per cent.

\subsection{Power density spectrum}

We compute the power density spectrum (PDS) from the background-subtracted light curves using the EPIC-pn data. We follow the procedure given in Heil et al. (2009) to construct the PDS. We fill the telemetry gaps ( $>15 \mathrm{~s}$ ) and gaps due to the short flares with local averages. We use a bin size of $0.220 \mathrm{~s}$ (three times the temporal resolution) to construct the PDS. We divide the entire light curve into intervals of 256 bins (56.32 s) and compute the PDS for each interval independently. Then we co-add all the PDS and average them in a single frame. The final PDS is rebinned geometrically in frequency space by a factor of 1.04 .

Fig. 2(a) and (d) show the Leahy power spectra (0.3-10.0 keV) for both observations (Obs-1 \& Obs-2). The PDS of Obs-1 (Fig. 2a) shows a QPO at centroid frequency $\sim 642 \mathrm{mHz}$. The PDS of Obs-2 (Fig. 2d) does not show any signature of a QPO-like feature. The PDS in the 0.3-5.0 keV range shows a QPO at $653 \mathrm{mHz}$ (Fig. 2b). 

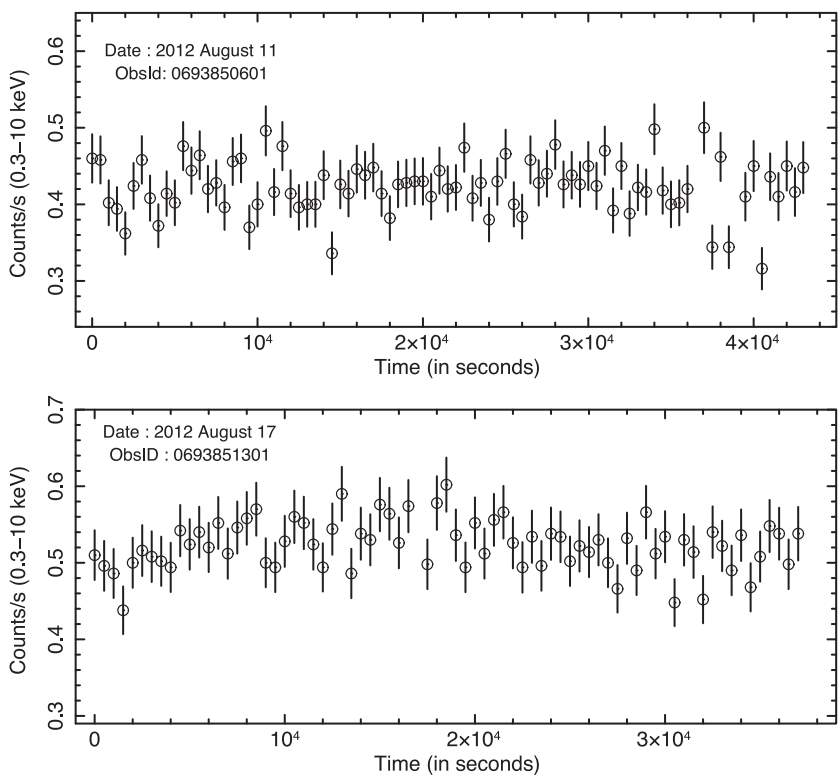

Figure 1. Photon-count variation of IC $342 \mathrm{X}-1$ observed with $X M M$ Newton (EPIC-pn data) in the energy band $0.3-10.0 \mathrm{keV}$. Top panel: the start time of observation was (Obs-1) 2012-08-11 20h 30m 47s (UT). Bottom panel: the start time of observation was (Obs-2) 2012-08-17 20h 12m 45s (UT). Each data point corresponds to a 500-s time bin.

However, no QPO is observed in the $5.0-10.0 \mathrm{keV}$ power spectrum (Fig. 2c).

We fit the PDS in the $0.3-10.0$ and $0.3-5.0 \mathrm{keV}$ bands (for Obs-1) with a model composed of a Lorentzian for a QPO peak, a power law $\left(\mathrm{AE}^{-\alpha}\right.$, where $\alpha=1.5 \pm 0.8$ for the $0.3-10.0 \mathrm{keV}$ band and $\alpha=1.95 \pm 0.9$ for the $0.3-5.0 \mathrm{keV}$ band) and a constant to account for the Poissonian noise. We obtain $\chi_{\text {red }}^{2}=1.02\left(\chi^{2} /\right.$ d.o.f. $\left.=45 / 44\right)$ for the $0.3-10.0 \mathrm{keV}$ band and $\chi_{\mathrm{red}}^{2}=1.06\left(\chi^{2} /\right.$ d.o.f. $\left.=47 / 44\right)$ for the $0.3-5.0 \mathrm{keV}$ band. The resultant fits are shown in Fig. 2(a) and (b) respectively. Fitting the power spectra (Obs-1) in the $0.3-10$ and $0.3-5.0 \mathrm{keV}$ bands with a power law and a constant gives $\chi_{\text {red }}^{2}=1.51$ $\left(\chi^{2} /\right.$ d.o.f. $\left.=71 / 47\right)$ and $\chi_{\text {red }}^{2}=1.57\left(\chi^{2} /\right.$ d.o.f. $\left.=74 / 47\right)$, respectively. Therefore, the overall fitting is improved upon considering the Lorentzian component for the QPO feature. Fitting the power spectra of Obs-2 in the 0.3-10.0 and 0.3-5.0 keV bands with a power law and a constant gives $\chi_{\text {red }}^{2}=1.22\left(\chi^{2} /\right.$ d.o.f. $\left.=57 / 47\right)$ and $\chi_{\text {red }}^{2}=1.02\left(\chi^{2} /\right.$ d.o.f. $\left.=48 / 47\right)$, respectively. We note that no Lorentzian component is required to improve the fit of the PDS of Obs- 2 in both energy bands (see Fig. $2 \mathrm{~d}$ and e). We also note that the PDS in the 5.0-10.0 keV energy bands for both observations (Obs-1 and Obs-2) are featureless and can be fitted with a constant to account for the Poissonian noise (see Fig. 2c and f). The significance of the QPO parameters in both bands is estimated with $F$-test statistics. The best-fitting model in the $0.3-10.0 \mathrm{keV}$ band gives a QPO of centroid frequency $v_{\mathrm{QPO}}=642 \pm 20 \mathrm{mHz}$, a $Q$ factor $(v / F W H M)=11.6$ (where FWHM is the full width at half-maximum) and an amplitude (root-mean-square) of 4.1 per cent with significance 3.6 $\sigma$. The best-fitting QPO parameters in the 0.3 $5.0 \mathrm{keV}$ band are centroid frequency $v_{\mathrm{QPO}}=653 \pm 30 \mathrm{mHz}, Q$ factor $=9.8$ and amplitude $(\mathrm{rms})=4.5$ per cent. The QPO in the $0.3-5.0 \mathrm{keV}$ band is detected with a significance of $3.7 \sigma$. The total integrated power $(0.01-2.5 \mathrm{~Hz})$ is $\sim 7.2$ per cent in the energy band $0.3-10.0 \mathrm{keV}$ for Obs-1 and 3.2 per cent for Obs-2. All errors quoted are computed using $\Delta \chi^{2}=1.0$.

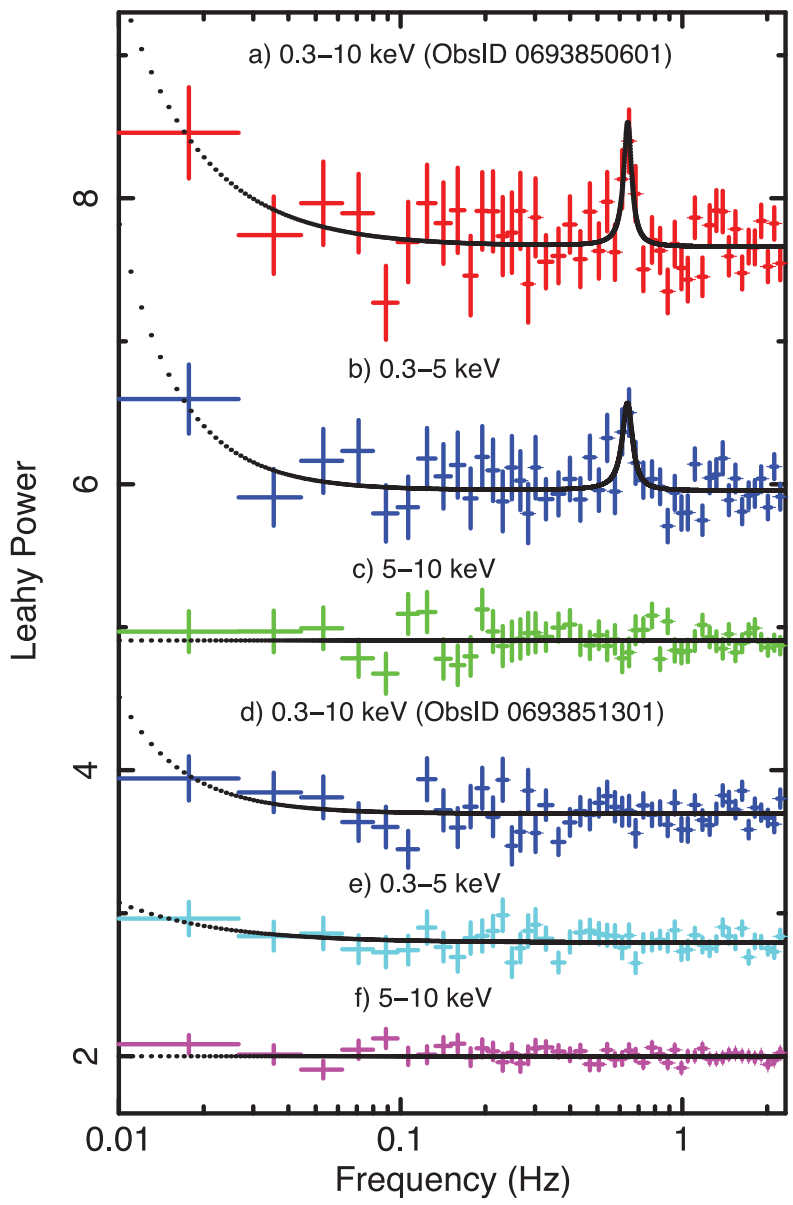

Figure 2. (a) The Leahy normalized power-density spectrum for Obs-1 in the $0.3-10.0 \mathrm{keV}$ band computed using EPIC-pn data. The PDS has been fitted with a Lorentzian for the QPO feature, a power law and a constant to account for Poissonian noise. The Poissonian noise has not been subtracted. (b) The Leahy normalized power-density spectrum for Obs-1 in the 0.3 $5.0 \mathrm{keV}$ band. A clear QPO feature fitted with a Lorentzian is seen. (c) The Leahy normalized power-density spectrum for Obs-1 in the $5.0-10.0 \mathrm{keV}$ band. (d) The Leahy normalized power-density spectrum for Obs-2 in the 0.3-10.0 keV band without any signature of a QPO-like feature. (e) The Leahy normalized power-density spectrum for Obs-2 in the $0.3-5.0 \mathrm{keV}$ band. (f) The power spectrum for Obs- 2 in the $5.0-10.0 \mathrm{keV}$ band. See text for details.

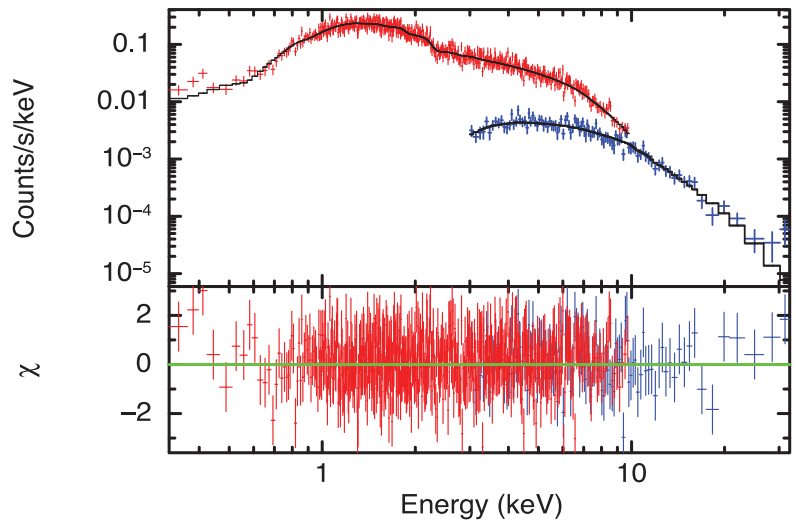

Figure 3. The combined EPIC-pn (for Obs-1) and NuSTAR/FPMA (2012 August 10) spectrum and folded model (top panel). The spectrum has been modelled with an absorbed diskbb plus cutoffpl model. The residual in units of sigma is shown in the bottom panel. 
Table 1. Summary of the spectral fits ( $\Gamma$ is photon index, $E_{\mathrm{c}}$ is cut-off energy, $k T_{\mathrm{in}}$ is disc temperature, $N_{\mathrm{dbb}}$ is disc normalization, $k T_{\mathrm{e}}$ is electron temperature of the corona and $\tau$ is optical depth of the corona. $F_{\text {tot }}$ is total flux in units of $10^{-12} \mathrm{erg} \mathrm{s}^{-1} \mathrm{~cm}^{-2}$ and $L_{\mathrm{tot}}$ is total luminosity in units of $10^{39} \mathrm{erg} \mathrm{s}{ }^{-1}$ ). Errors quoted are calculated at 68 per cent confidence level.

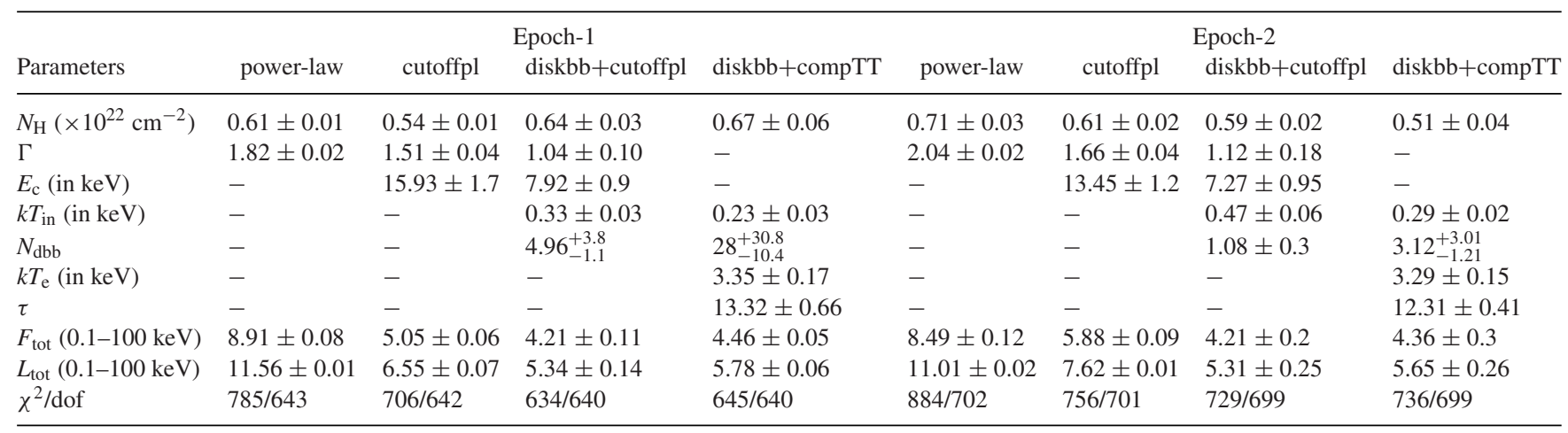

Note that considering the time interval (Obs-1) before the short flares results in $36.5 \mathrm{ks}$ continuous observation. The PDS created using this exposure time ( $36.5 \mathrm{ks}$ ) shows a QPO feature with similar parameters. However, the significance of the QPO changes from 3.6 to $3.4 \sigma$.

\subsection{Energy spectrum}

The energy spectra of EPIC-pn and NUSTAR/FPMA are analysed using XSPEC version 12.8.1. We fit the combined EPIC-pn (0.3-10.0 keV band; Obs-1) and NuSTAR/FPMA (3.0-40.0 keV; 2012 August 10) data (epoch-1) with the following models:

(1) a power law,

(2) a power law with exponential cut-off (cutoffpl in XSPEC),

(3) cutoffpl, with an addition of a multi-temperature disc component for the standard thin accretion disc (diskbb in XSPEC: Mitsuda et al. 1984) and

(4) diskbb plus Comptonization model (compTT model of XSPEC: Titarchuk 1994).

We consider the tbabs model (Wilms, Allen \& McCray 2000) for all spectral models, in order to model the extinction $\left(N_{\mathrm{H}}\right)$ on the line of sight to the source. Similarly, the second quasi-simultaneous data (epoch-2) obtained with EPIC-pn (0.3-10.0 keV; Obs-2) and NuSTAR/FPMA (3.0-40.0 keV; 2012 August 16) are also analysed and fitted with the above-mentioned models. The cross-calibration constant between NuSTAR/FPMA and XMM-Newton/EPIC-pn is found to be close to $1(\sim 0.95)$.

The best-fitting parameters for all models are listed in Table 1. All errors quoted are computed using $\Delta \chi^{2}=1.0$ (at 68 percent confidence). The $N_{\mathrm{H}}$ values are found to be in the range 0.5$0.7 \times 10^{22} \mathrm{~cm}^{-2}$ (see Table 1). For epoch-1, the power-law and cutoffpl models give $\chi_{\text {red }}^{2}=1.22\left(\chi^{2} /\right.$ d.o.f. $\left.=785 / 643\right)$ and $\chi_{\text {red }}^{2}=1.10$ $\left(\chi^{2} /\right.$ d.o.f. $\left.=706 / 642\right)$, respectively. The probability that the fit is improved by chance is $1.59 \times 10^{-16}$. Hence a simple power-law model is not the best description of the data and the spectrum shows a clear high-energy cut-off. Since the soft excess modelled with diskbb has been observed in many ULXs (Miller et al. 2003, 2004; Feng \& Kaaret 2005; Stobbart et al. 2006), we fitted the epoch-1 spectrum with the diskbb+cutoffpl model. The fit yields $\chi_{\text {red }}^{2}=0.99\left(\chi^{2} /\right.$ d.o.f. $\left.=634 / 640\right)$ and the chance improvement probability equals $1.12 \times 10^{-15}$ for inclusion of the diskbb component, suggesting that a soft excess component below $2 \mathrm{keV}$ is required to improve the fit.
Analysis of XMM-Newton data of several ULXs revealed that disc emission plus a cool $\left(k T_{\mathrm{e}} \sim 3 \mathrm{keV}\right)$ and optically thick ( $\tau \sim 5-30$ ) Comptonized component models the spectral data well (Gladstone et al. 2009). Hence, we tried with the compTT model also, instead of cutoffpl. The combination of diskbb plus compTT model, where the seed photon temperature is tied to the inner disc temperature, gives $\chi_{\text {red }}^{2}=1.01\left(\chi^{2} /\right.$ d.o.f. $\left.=645 / 640\right)$. Hence, the diskbb+cutoffpl model is the best description for the epoch-1 observation. Epoch-2 data can also be modelled well with the diskbb+cutoffpl model, resulting in $\chi_{\text {red }}^{2}=1.04$ $\left(\chi^{2} /\right.$ d.o.f. $\left.=729 / 699\right)$.

The disc temperature is $0.33 \pm 0.03$ and $0.47 \pm 0.06 \mathrm{keV}$, respectively, for the epoch-1 and epoch-2 data. The diskbb normalization is found to be unphysically small for both spectra. The cut-off energy $\left(E_{\mathrm{c}}\right)$ is $7.92 \pm 0.91$ and $7.27 \pm 0.99 \mathrm{keV}$, respectively, for epoch-1 and epoch-2 spectra (see also Rana et al. 2014). Although the diskbb+cutoffpl model is the best description of the spectra, the diskbb+compTT model also provides a statistically good description of the spectra for both epochs. The best-fitting optical depth $(\tau)$ and electron temperature $\left(k T_{\mathrm{e}}\right)$ for the epoch- 1 spectrum are $13.32 \pm 0.66$ and $3.35 \pm 0.17 \mathrm{keV}$, respectively. The bestfitting $\tau$ and $k T_{\mathrm{e}}$ for the epoch-2 spectrum are $12.31 \pm 0.41$ and $3.29 \pm 0.15 \mathrm{keV}$, respectively.

In Table 1, we also give the estimated unabsorbed total flux in the $0.1-100.0 \mathrm{keV}$ band and the corresponding luminosities for all models using a distance of $3.3 \mathrm{Mpc}$. The unabsorbed fluxes and corresponding errors are computed using the convolution model cflux of XSPEC.

\section{DISCUSSION AND SUMMARY}

In the present work, we report the discovery of a QPO in the XMMNewton data for the ULX source IC 342 X-1, a system harbouring a black hole (see Okada et al. 1998). The detection of a peak at $\sim 642 \mathrm{mHz}(Q \sim 11.6, \mathrm{rms} \sim 4.1$ per cent and significance $3.6 \sigma)$ in the power spectrum of IC $342 \mathrm{X}-1$ (Obs-1) could be used to constrain the mass of the 'hole', as this technique was employed for other ULXs (Dewangan et al. 2006a; Strohmayer et al. 2007; Rao et al. 2010). Interestingly, the QPO detection in IC 342 X-1 is also the highest frequency observed in a ULX to date, whereas the subsequent $X M M-$ Newton observation ( $6 \mathrm{~d}$ later) does not show any signature of a QPO-like feature.

Detailed spectral analysis shows that the broad-band spectrum $(0.3-40.0 \mathrm{keV})$ of the source (epoch-1) is well described by an absorbed diskbb plus a cutoffpl or a diskbb plus a compTT model. 
The best-fitting model parameters indicate that the source was in a hard spectral state $\left(\Gamma \sim 1.04, \tau \sim 13.32, E_{\mathrm{c}} \sim 7.92 \mathrm{keV}\right)$ that is unlike the canonical hard state as commonly seen in Galactic black hole binaries (GBHBs). The unabsorbed luminosity of the source is found to be around $\sim 5.34 \times 10^{39} \mathrm{erg} \mathrm{s}^{-1}$. Similar spectral features are also seen in the epoch- 2 observation. All these observational results are consistent with the findings of Gladstone et al. (2009) and imply that the source could be in the hard ultraluminous state (Sutton et al. 2013).

In general, the power spectra of Galactic black hole (GBH) sources are characterized by various broad noise components (power-law-like red noise, flat-top noise etc.) along with a narrow noise component (i.e. Lorentzian type for a QPO-like feature). The high temporal variability and low-frequency QPOs $(\sim 0.1-20 \mathrm{~Hz})$ observed in $\mathrm{BH}$ sources are mostly associated with hard or intermediate states. In general, the low-frequency QPOs seen in Galactic BHs are classified as A, B and C type (Casella, Belloni \& Stella 2005). As an example, a C-type QPO has a high $Q$ factor (6-12), a large amplitude (3-16 percent rms) and shows complex phase lag behaviour along with a flat-top noise component. Moreover, low-frequency QPOs evolve with the spectral state and disappear completely in the thermally dominated soft state (Remillard \& McClintock 2006; Nandi et al. 2012). Since QPOs scale inversely with the mass of the black hole in GBH sources (Shrader \& Titarchuk 2003; Shaposhnikov \& Titarchuk 2009), the observed QPOs can be used to infer the mass of the putative black hole.

In contrast, the nature of the power spectra and the short-term variability are more complex in ULXs (Heil et al. 2009). Few sources show signatures of QPOs without any change in the centroid QPO frequency (except M82 X-1, see Feng \& Soria 2011 for details). The recent discovery of twin-peak X-ray QPOs (3:2 frequency ratio) in M82 X-1 is found to be stable (see Pasham et al. 2014). No definite correlation exists between QPOs and spectral states in ULXs, but a defined correlation exists for most GBHBs. In the case of IC 342 X-1, the power spectrum of Obs-1 can be modelled with a constant and a Lorentzian feature (for the QPO), along with a power-law component. The observed intrinsic variability is around $\sim 7.2$ percent. This form of PDS is a common characteristic of ULXs (see Heil et al. 2009), where significant variability is observed and the power spectra are modelled with a power law or with a broken power-law component. Interestingly, the power spectrum of Obs-2 (6 d later) does not show any presence of a QPO, but both observations (Obs-1 \& Obs-2) show similar spectral nature (see Table 1).

Another important thing to note is that the hard X-ray energy spectra of GBHBs (i.e. hard or intermediates states) show a connection with the strong QPOs and compact radio jets observed in the sources (Radhika \& Nandi 2014). The high-energy spectral curvature observed in GBHBs (in the range $30-100 \mathrm{keV}$ ) is the manifestation of an optically thin corona with a characteristic temperature of $\sim 100 \mathrm{keV}$ (Remillard \& McClintock 2006). However, in ULXs, the spectral nature is quite different, with a spectral curvature of the electron temperature of a few $\mathrm{keV}$ and an optical depth in the range 5-30 (thick corona). Also, the connection between the hard X-ray spectral nature, QPOs and compact jets is not well established in ULXs. It is therefore hard to compare the spectral nature of ULXs with that of GBHBs.

Since the characteristic time-scale of an active galactic nucleus (AGN) or GBH scales with the compact object mass (McHardy et al. 2006), one can raise the following basic question: could the observed QPO in IC 342 X-1 be analogous to any type of QPO observed in Galactic black holes? There have been several attempts to identify the QPOs seen in ULXs (Strohmayer \& Mushotzky 2009; Pasham \& Strohmayer 2012; Feng et al. 2010) with those seen in GBHBs. However, the classification of QPOs observed in ULXs remains unclear (Middleton et al. 2011). The detection of a 642-mHz QPO in IC $342 \mathrm{X}-1$ that shows some of the characteristics ( $Q$ factor $=11.6$ and $\mathrm{rms}=4.1$ per cent) of a C-type QPO can be used to constrain the black hole mass in IC 342 X-1. However, a true classification requires a phase-lag study, which is beyond the scope of the present work. The heaviest Galactic BH source GRS 1915+105 (Greiner, Cuby \& McCaughrean 2001) shows C-type QPOs in the frequency range $1-3 \mathrm{~Hz}$. If we consider that the $642-\mathrm{mHz}$ QPO is a scaled-down version of the QPOs seen in GRS 1915+10, along with the assumption that QPO frequencies scale inversely proportional to BH masses (Remillard \& McClintock 2006), then we can estimate the black hole mass in IC $342 \mathrm{X}-1$ as $M_{\mathrm{BH}}$ (IC342X1) $\sim v_{\mathrm{QPO}}(\mathrm{GRS} 1915) / v_{\mathrm{QPO}}(\mathrm{IC} 342 \mathrm{X}-1) \times M_{\mathrm{BH}}(\mathrm{GRS} 1915) \sim 20$ $65 \mathrm{M}_{\odot}$ (considering the mass of GRS $1915+105 \sim 14 \mathrm{M}_{\odot}$ ). It implies that, for the bolometric luminosity $\sim 5.34 \times 10^{39} \mathrm{erg} \mathrm{s}^{-1}$, the black hole at the centre of IC $342 \mathrm{X}-1$ is accreting matter at near-Eddington rate $\left(\sim 0.7-2 L_{\text {Edd }}\right)$.

Another possibility for estimating the mass of the black hole in IC $342 \mathrm{X}-1$ is to consider the observed QPO as a scaled-down version of those high frequency QPOs (HFQPOs) that are observed in GBHBs (e.g. 65-67 Hz in GRS 1915+105, see Morgan, Remillard \& Greiner 1997; $66 \mathrm{~Hz}$ in IGR J17091-3624, see Altamirano \& Belloni 2012). In this scenario, the estimated mass could be in the range $\sim 1000-1800 \mathrm{M}_{\odot}$ (considering the mass of GRS $\left.1915+105 \sim 14 \pm 4 \mathrm{M}_{\odot}\right)$. This would imply that the central black hole accretes matter at a sub-Eddington rate $\left(0.02-0.05 L_{\text {Edd }}\right)$. This may not be the case for IC $342 \mathrm{X}-1$, as the observed spectral properties favour the hard ultraluminous state of ULXs, explained by a system harbouring a stellar-mass black hole accreting at and above the Eddington limit (Gladstone et al. 2009; Sutton et al. 2013).

We are then left with the possibility that the central 'hole' of IC 342 X-1 might be harbouring a 'massive' stellar-mass black hole of mass $\sim 20-65 \mathrm{M}_{\odot}$. The present stellar evolution models also predict massive $\mathrm{BH}$ remnants $\left(\sim 20-100 \mathrm{M}_{\odot}\right)$ that could be formed from the direct collapse of the progenitor without any supernova explosion (Fryer 1999).

\section{ACKNOWLEDGEMENTS}

The authors thank the anonymous referee for a careful reading of the article and constructive criticism, along with useful suggestions for improvement of the manuscript. We also thank Drs Girish and Das for careful reading and comments on the manuscript. This research has made use of data and/or software provided by the High Energy Astrophysics Science Archive Research Center (HEASARC). We thank Dr Anil Agarwal, GD, SAG, Mr Vasantha E DD, CDA and Dr S. K. Shivakumar, Director, ISAC, for encouragement and continuous support to carry out this research.

\section{REFERENCES}

Altamirano D., Belloni T., 2012, ApJ, 747, L4

Bachetti M. et al., 2014, Nat, 514, 202

Begelman M. C., 2002, ApJ, 568, L97

Begelman M. C., King A. R., Pringle J. E., 2006, MNRAS, 370, 399

Bregman J. N., Cox C. V., Tomisaka K., 1993, ApJ, 415, L79

Casella P., Belloni T., Stella L., 2005, ApJ, 629, 403

Colbert E. J. M., Mushotzky R. F., 1999, ApJ, 519, 89

Cseh D. et al., 2012, ApJ, 749, 17 
Dewangan G. C., Titarchuk L., Griffiths R. E., 2006a, ApJ, 637, L21

Dewangan G. C., Griffiths R. E., Rao A. R., 2006b, ApJ, 641, L125

Fabbiano G., 1988, ApJ, 325, 544

Fabbiano G., 1989, ARA\&A, 27, 87

Fabbiano G., Trinchieri G., 1987, ApJ, 315, 46

Feng H., Kaaret P., 2005, ApJ, 633, 1052

Feng H., Soria R., 2011, New Astron. Rev., 55, 166

Feng H., Rao F., Kaaret P., 2010, ApJ, 710, L137

Fryer C. L., 1999, ApJ, 522, 413

Gladstone J. C., Roberts T. P., Done C., 2009, MNRAS, 397, 1836

Greiner J., Cuby J. G., McCaughrean M. J., 2001, Nat, 414, 522

Heil L. M., Vaughan S., Roberts T. P., 2009, MNRAS, 397, 1061

King A. R., 2002, MNRAS, 335, L13

Kubota A., Mizuno T., Makishima K., Fukazawa Y., Kotoku J., Ohnishi T., Tashiro M., 2001, ApJ, 547, L119

Liu J., Bregman J. N., Bai Y., Justham S., Crowther P., 2013, Nat, 503, 500

Long K. S., van Speybroeck L. P., 1983, in Lewin W. H. G., van den Heuvel E. P. J., eds, Accretion-Driven Stellar X-ray Sources. Cambridge Univ. Press, Cambridge, p. 117

Marlowe H. et al., 2014, MNRAS, 444, 642

McHardy I. M., Koerding E., Knigge C., Fender R. P., 2006, Nat, 444, 730

Middleton M. J., Roberts T. P., Done C., Jackson F. E., 2011, MNRAS, 411, 644

Miller J. M., Fabbiano G., Miller M. C., Fabian A. C., 2003, ApJ, 585, L37

Miller J. M., Fabian A. C., Miller M. C., 2004, ApJ, 607, 931

Mitsuda K. et al., 1984, PASJ, 36, 741

Morgan E. H., Remillard R. R., Greiner J., 1997, ApJ, 482, 993

Motch C., Pakull M., Soria R., Grise F., Pietrzynski G., 2014, Nat, 514, 198

Nandi A., Debnath D., Mandal S., Chakrabarti S. K., 2012, A\&A, 542, 56
Okada K., Dotani T., Makishima K., Mitsuda K., Mihara T., 1998, PASJ, 50,25

Pasham D. R., Strohmayer T. E., 2012, ApJ, 753, 139

Pasham D. R., Strohmayer T. E., Mushotzky R. F., 2014, Nat, 513, 74

Pintore F., Zampieri L., Wolter A., Belloni T., 2014, MNRAS, 439, 3461

Radhika D., Nandi A., 2014, Adv. Space Res., 54, 1678

Rana V. et al., 2014, ApJ, preprint (arXiv:1401.4637)

Rao F., Feng H., Kaaret P., 2010, ApJ, 722, 620

Remillard R. A., McClintock J. E., 2006, ARA\&A, 44, 49

Reynolds C. S., Loan A. J., Fabian A. C., Makishima K., Brandt W. N., Mizuno T., 1997, MNRAS, 286, 349

Roberts T. P., 2007, Ap\&SS, 311, 203

Roberts T. P., Warwick R. S., 2000, MNRAS, 315, 98

Saha A., Claver J., Hoessel J. G., 2002, AJ, 124, 839

Shaposhnikov N., Titarchuk L., 2009, ApJ, 699, 453

Shrader C. R., Titarchuk L., 2003, ApJ, 598, 168

Stobbart A.-M., Roberts T. P., Wilms J., 2006, MNRAS, 368, 397

Strohmayer T. E., Mushotzky R. F., 2003, ApJ, 586, L61

Strohmayer T. E., Mushotzky R. F., 2009, ApJ, 703, 1386

Strohmayer T. E., Mushotzky R. F., Winter L., Soria R., Uttley P., Cropper M., 2007, ApJ, 660, 580

Sutton A. D., Roberts T. P., Middleton M. J., 2013, MNRAS, 435, 1758

Titarchuk L., 1994, ApJ, 434, 570

Wilms J., Allen A., McCray R., 2000, ApJ, 542, 914

Yoshida T., Isobe N., Mineshige S., Kubota A., Mizuno T., Saitou K., 2013, PASJ, 65, 48

This paper has been typeset from a $\mathrm{T}_{\mathrm{E}} \mathrm{X} / \mathrm{L} \mathrm{T} \mathrm{E} \mathrm{X}$ file prepared by the author. 